\title{
An Electrophysiological Correlate of Visual Motion Awareness in Man
}

\author{
Thomas Haarmeier and Peter Thier \\ University of Tübingen
}

\begin{abstract}
It is usually held that perceptual spatial stability, despite smooth pursuit eye movements, is accomplished by comparing a signal reflecting retinal image slip with an internal reference signal, encoding the eye movement. The important consequence of this concept is that our subjective percept of visual motion reflects the outcome of this comparison rather than retinal image slip. In an attempt to localize the cortical networks underlying this comparison and therefore our subjective percept of visual motion, we exploited an imperfection inherent in it, which results in a movement illusion. If smooth pursuit is carried out across a stationary background, we perceive a tiny degree of illusionary background motion (Filehne illusion, or FI), rather than experiencing the ecologically optimal percept of stationarity. We have recently shown that this
\end{abstract}

\section{INTRODUCTION}

To maintain a sense of perceptual spatial stability despite self-motion is a prerequisite for successful spatial orientation. Toward this end, the brain has to discriminate sensory signals resulting from the observer's own activities from those arising from the external world. For instance, retinal slip of the image of a stationary object resulting from self-motion such as slow-tracking eye movements should not be mistaken as motion of the same object in the external world. Following early suggestions by von Helmholtz (1910), it is usually held that spatial stability is the consequence of the fact that a signal reflecting retinal image slip is compared with an internal reference signal, encoding the eye movement (von Holst \& Mittelstaedt, 1950; Sperry, 1950; Wertheim, 1994). An important implication of this concept is that our subjective percept of visual motion reflects the outcome of this comparison rather than retinal image slip. In an attempt to localize the cortical networks underlying this comparison and therefore our subjective percept of visual motion, we exploited an imperfection inherent in it, which results in a movement illusion. If smooth pursuit is carried out across a stationary background, we perceive a tiny degree of illusionary background motion (Filehne illusion, or FI), rather than experiencing the ecologically optimal percept of station-

arity. According to the original description by Filehne (1922), this illusionary motion is directed opposite to the eye movement being executed. Later studies also demonstrated inverted FIs and, more generally, pointed toward a profound dependence of the size and direction of the FI on the specific details of the visual configuration prevailing (de Graaf \& Wertheim, 1988; Wertheim, $1981,1987,1994)$. We have recently shown that the illusion can, moreover, be modified substantially and predictably under laboratory conditions by visual motion unrelated to the eye movement (Haarmeier \& Thier, 1996). In the present event-related potential (ERP) study we made use of the fact that identical constellations of retinal image slip and eye movement can yield different percepts of illusionary background motion in order to locate the neuronal substrate underlying the comparison of the two neuronal signals. Toward this end, ERPs obtained under two conditions, which were identical physically but gave rise to very different FIs, were compared.

ERP recordings were obtained from 10 naive subjects who performed smooth pursuit eye movements across a stationary background (ERP trials, Figure 1). Pursuit was elicited by a small target moving at $12 \% \mathrm{sec}$ rightward on an otherwise dark computer monitor. Temporally located in the middle of the target sweep, when the eyes were close to straight ahead, a stationary background pattern was presented for $300 \mathrm{msec}$. Subjects 
Figure 1. Course of an ERP trial. The subject performs smooth pursuit eye movements by tracking a small dot that travels at $12^{\circ} / \mathrm{sec}$ to the right. While the eyes are close to straight ahead, a stationary background is presented for $300 \mathrm{msec}$ (gray column). The subject is instructed to maintain pursuit and to report the direction of perceived background motion by pressing one of two alternative keys (horizontal bar). Inset: ERP triggered $300 \mathrm{msec}$ prior to the presentation of the background.

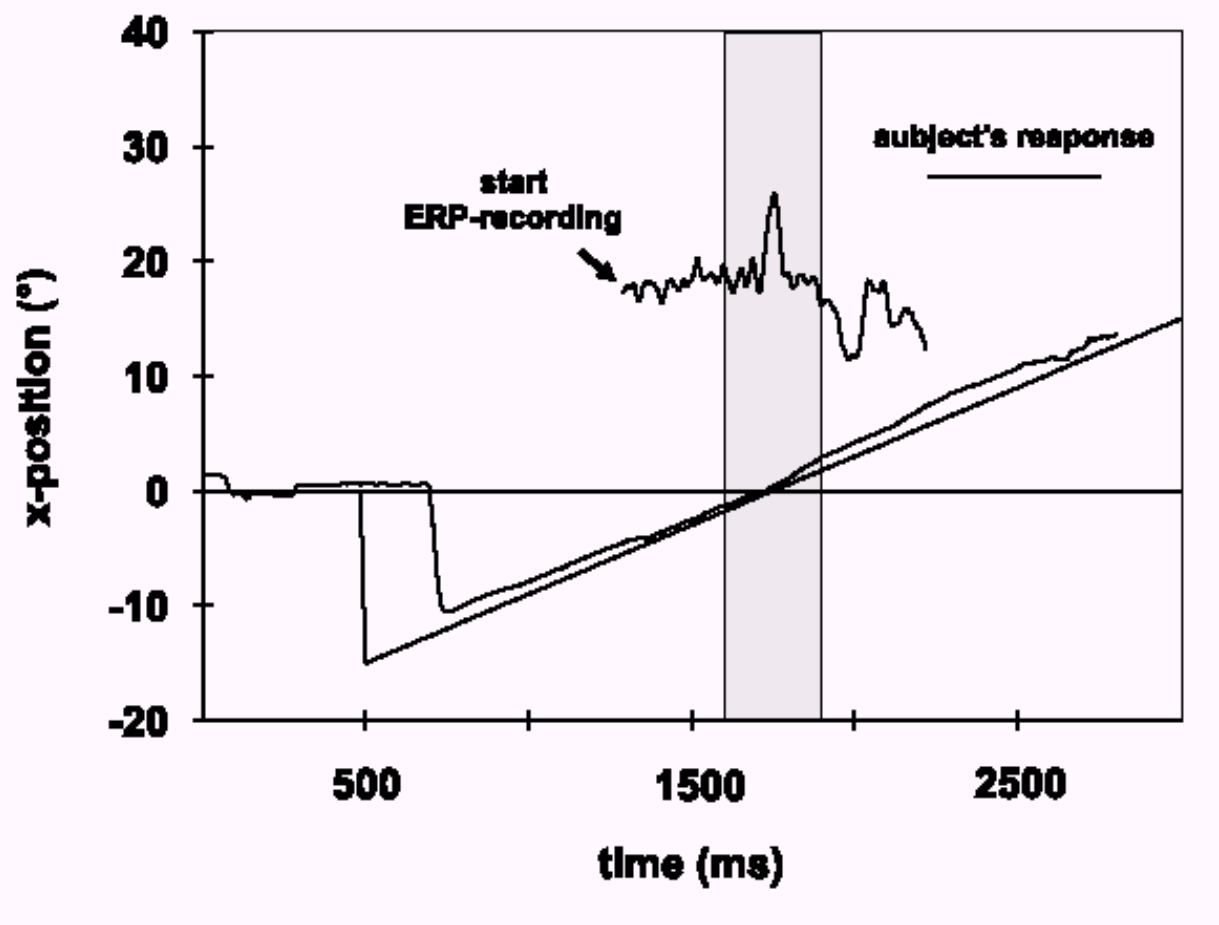

were asked to report what direction of background movement they experienced during pursuit by pressing one of two alternative keys related to leftward and rightward background movement, respectively.

In order to correlate the subjects' perception of the velocity of the stationary background with the potentials obtained in the ERP trials, it was necessary to exactly determine the size of the Filehne illusion. Toward this end, ERP trials were presented randomly interleaved with a second kind of trial (PEST trials, Parameter Estimation by Sequential Testing). In these PEST trials the background stimuli presented moved en bloc at a horizontal velocity, determined by an adaptive staircase procedure (Taylor \& Creelman, 1967) in order to pinpoint the amount of external background motion required to compensate for the FI, thus yielding the impression of a stationary background (Mack \& Herman, 1973, 1978). At this point of subjective stationarity (PSS) background stimulus velocity is equal in magnitude but opposite in direction to the illusionary motion perceived during the ERP trials.

Whereas ERP stimuli were used to collect electrophysiological recordings, and PEST stimuli to measure the Filehne illusion, a third stimulus class, presented randomly interleaved with the two previous ones, was designed to create two perceptually distinct conditions. In these conditioning stimulus (CS) trials the background moved at a constant and high horizontal velocity. As recently shown by us (Haarmeier \& Thier, 1996), the perception of pursuit-related background motion can be influenced by such conditioning stimuli in a predictable way. In this study each subject participated in two experimental sessions that differed in the velocity of the $\mathrm{CS}$, which was chosen such as to yield a high FI in one condition (velocity of $\mathrm{CS}=-12^{\circ} / \mathrm{sec}$, high FI condition) or, alternatively, to yield a low FI in the other one (velocity of $\mathrm{CS}=+8^{\circ} / \mathrm{sec}$, low FI condition). The principal effect of the conditioning stimuli on the Filehne illusion can be drawn from Figure 2, which shows the temporal sequence of a typical measurement under both conditions. When the conditioning stimuli moved in the same direction as the eyes (lower panel), the motion illusion evoked by pursuit across the stationary background in the ERP trials was almost negligible as indicated by the fact that the sequence of PEST trials converges toward $0 \% \mathrm{sec}$. Conversely, presentation of conditioning stimuli directed opposite to the movement of the eyes resulted in a deflection of the PEST stimuli toward large, negative values (here: $-4^{\circ} / \mathrm{sec}$, upper panel), equivalent to an increased Filehne illusion in the ERP trials. Comparing the potentials obtained under the low and high FI condition from the individual subjects will allow us to identify those components whose amplitudes are determined by the subjective perception of visual motion.

\section{RESULTS}

\section{Behavioral Results}

Background velocity at PSS averaged $-3.57 \% \mathrm{sec}$ in the high and $-0.91 \%$ sec in the low FI condition (Figure 3, 
Figure 2. Temporal sequence of background stimulus velocities that were presented to one of the subjects during the measurement under the high (upper panel) and the low (lower panel) Filehne illusion condition. Each dot marks one trial, intertrial interval $0.5 \mathrm{sec}$. Negative background velocities indicate direction opposite to eye movements.

Background stimuli presented in the ERP, PEST, and CS trials share the same physical properties but serve different purposes. Under both conditions ERP responses are only collected when a stationary background is presented. The background motion perceived during the ERP trials is quantified by means of PEST stimuli that converge toward the point of subjective stationarity (PSS). Conditioning stimuli at different velocity levels lead to a shift of the PSS and thus to different Filehne illusions in the ERP trials. Subjects were naive with respect to the different kinds of randomly interleaved background stimuli and always reported the direction of perceived background motion while tracking the pursuit target.

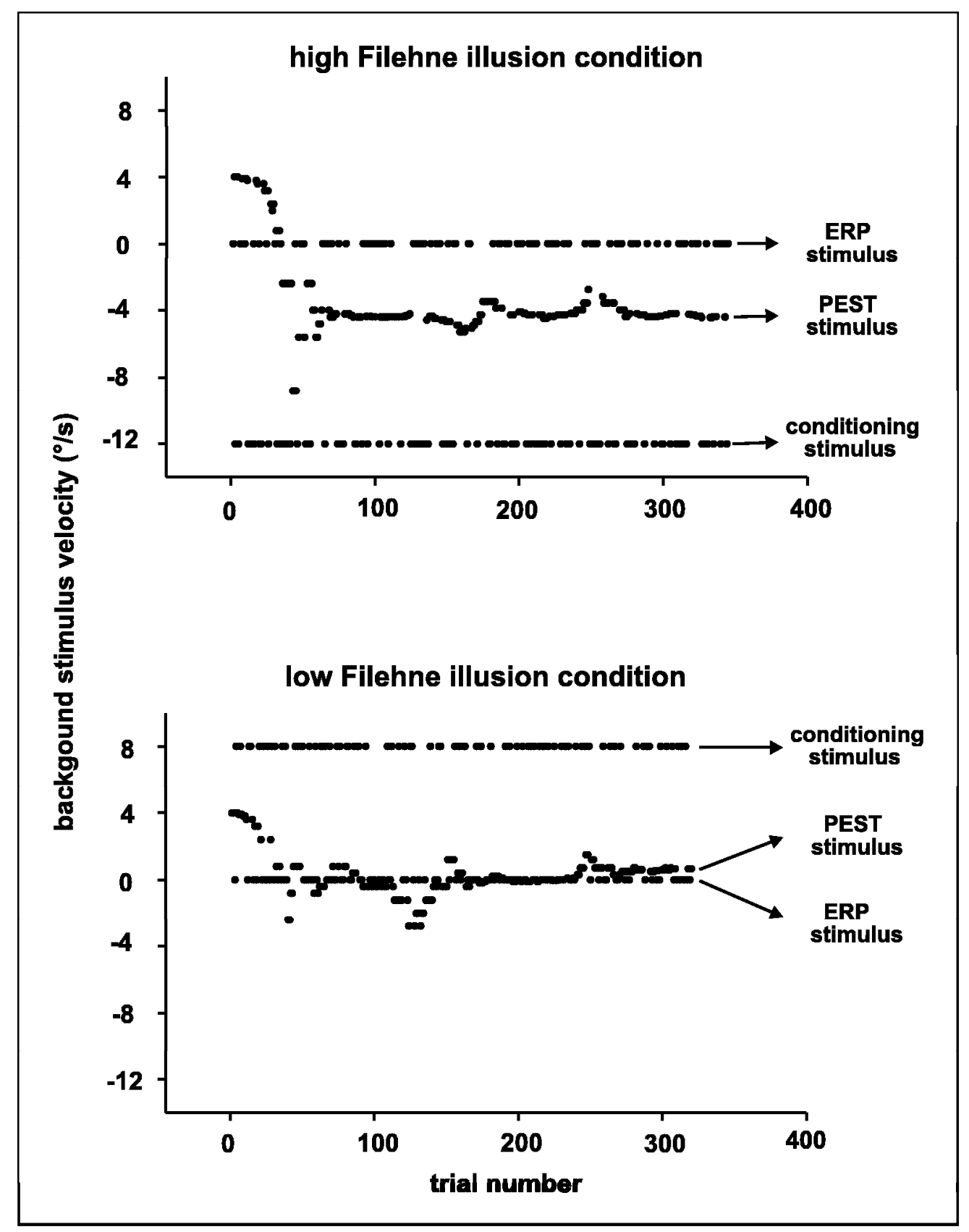

right panel). The difference of perceived background motion in the ERP trials, thus, averaged $2.66 \%$ sec and was statistically highly significant (paired $t$ test, $p<$ 0.001). This effect was not attributable to differences in oculomotor performance, which was shown to be invariant for the two sessions. Specifically, group means of eye velocity during background presentation for PEST trials were 9.97 and $9.82^{\circ} / \mathrm{sec}$ for the high and the low FI condition, respectively (paired $t$ test, $p>0.05$ ). The same was true for the eye movements during the ERP trials with the eye velocity averaging $9.86 \%$ sec in the low FI condition and $9.98^{\circ} / \mathrm{sec}$ in the other (paired $t$ test, $p>$ 0.05 ). Finally, there was no statistical difference in oculomotor performance during PEST trials as compared to the ERP trials, confirming that the results obtained from the psychophysical strategy can serve as a reliable estimate of the motion perceived during recording of the ERPs.

Despite the overall modification of the FI, the amount of modulation achieved varied considerably between subjects with differences of PSS between conditions ranging from 0.9 to $4.64^{\circ} / \mathrm{sec}$ (Figure 3, left panel). For further analysis subjects were therefore divided according to their degree of FI modulation. Two groups of five subjects each were formed, encompassing the five subjects showing the highest (Filehne-responder) and the smallest (Filehne-nonresponder) FI modification. 
Figure 3. Background stimulus velocity at the PSS (i.e. the estimate of the Filehne illusion) as a function of the velocity of the conditioning stimulus. Ten subjects, represented by individual symbols (left panel) and means/standard deviations (right panel). Negative velocities indicate direction opposite to the eye movement.

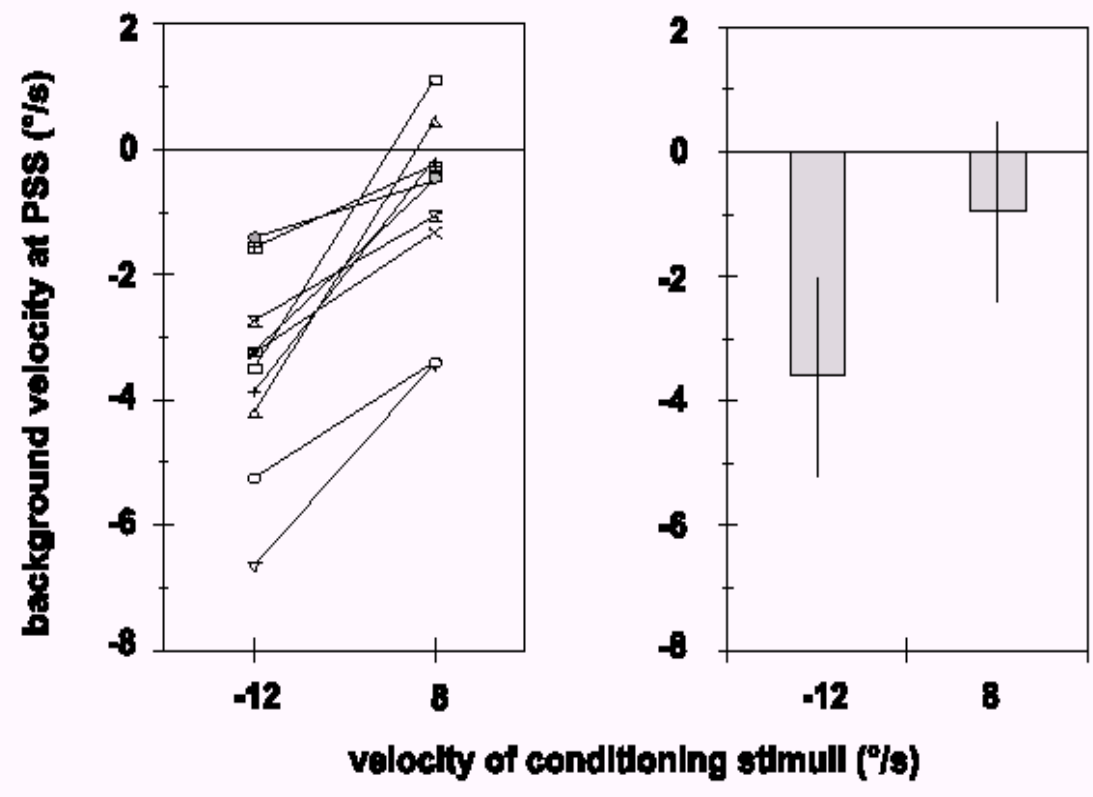

\section{Electrophysiological Results}

ERPs obtained under the low and the high FI condition exhibited the same principal pattern of components (Figure 4b). The earliest components were a first positive peak at $\sim 115 \mathrm{msec}$ (P1) and a first negative component at $\sim 180 \mathrm{msec}$ (N200). These potentials were followed by a second positivity (P2, peak latency $\sim 240 \mathrm{msec}$ ), a second negativity (at $\sim 300 \mathrm{msec}$ ) that we will refer to as the N300 component, and a large positivity (P300, at $\sim 380 \mathrm{msec}$ ). In order to determine those components related to the motion perceived we performed separate running $t$ tests on the difference between potentials obtained under the two conditions for the group of responders and nonresponders. At least 15 consecutive $t$ test values exceeding the 0.01 level of significance were defined as the critical statistical criteria for determining a differential effect (Rugg, Doyle, \& Well, 1995). This analysis revealed no significant difference in the group of Filehne-nonresponders (Figure 4a, right panel). This was also true for the early components of the ERP responses in the group of Filehne-responders (P1, N200, P2; Figure 4a, left panel; Figure $4 \mathrm{~b}$ ). The first significant effect was seen in the parietal and occipital leads of the Filehne-responders, all of them reaching significance at 292 to 294 msec, which marks the latency of the N300 component. The second effect was restricted to the frontal lead with highest $t$ values at $370 \mathrm{msec}$, the latency of the P300 (Figure $4 \mathrm{~b}, \mathrm{c}$ ). These results were confirmed when the differences of potentials were compared between groups. In order to assess in more detail the relation between peak amplitude modulation and change in motion perception, the differences of peak amplitude were measured in each individual subject for the electrodes where statistically significant effects were observed. Specifically, for the N300, individual means of amplitudes for all parietal and occipital leads were calculated, whereas the responses of the P300 were estimated for the frontal lead. For both potentials, we observed a strong correlation between psychophysical results and ERP responses with higher peaks for higher modulation of the Filehne illusion (Figure 5).

\section{DISCUSSION}

Our results provide a first electrophysiological correlate of visual stability in man. We made use of the fact that the retinal slip of the image of a stationary background induced by smooth pursuit eye movements can yield different percepts of background motion depending on the preceding visual stimulation. In a previous psychophysical study (Haarmeier \& Thier, 1996), we demonstrated that the modulation of the Filehne illusion is not attributable to biasing effects or to motion adaptation but that it might, instead, reflect the dynamic character of the networks elaborating spatial constancy. Similar phenomena have recently been reported for other kinds of self-motion such as running (Pelah \& Barlow, 1996) and have been interpreted in terms of what von Helmholtz (1910) referred to as unconscious inference. Recalibration of the mechanisms that estimate the associations between the sensory message and an internal representation of self-motion is most likely to account for these phenomena.

In the present study we observed two different poten- 


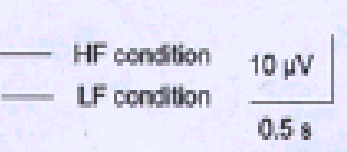

Fllehne-nonresponder
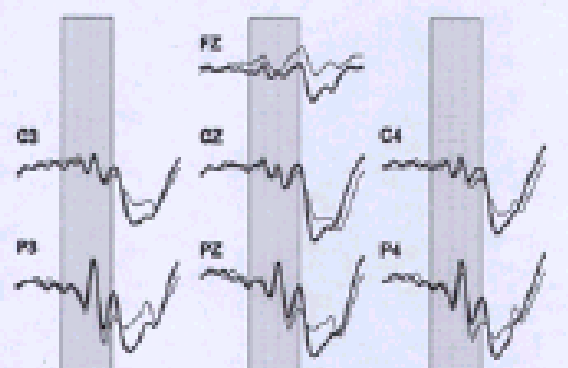

$\mathrm{Pz}$

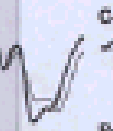
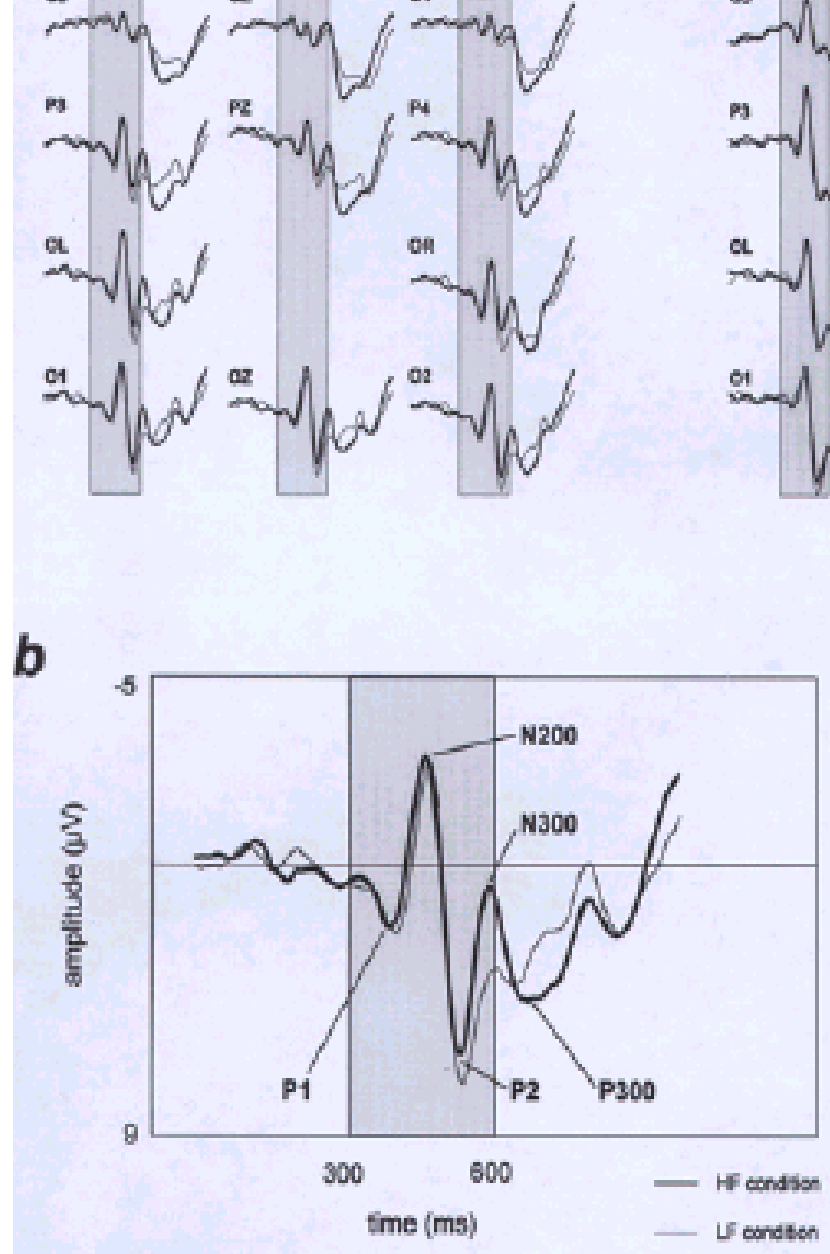

c

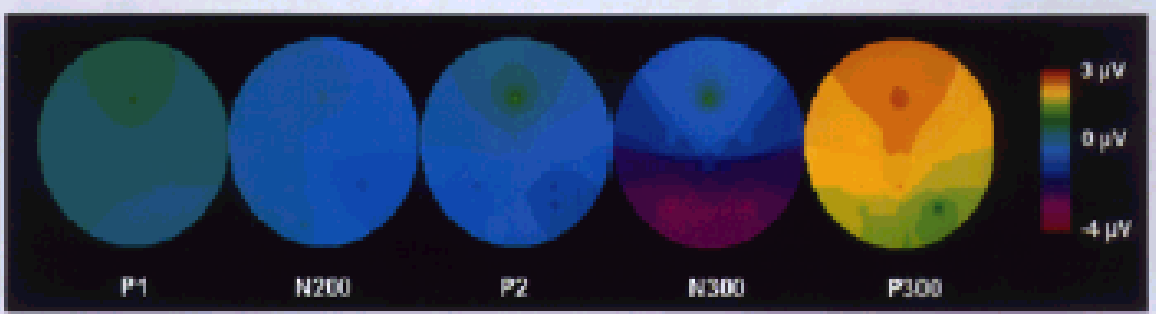

Figure 4. Mean ERP responses for the group of Filehne-responders and Filehne-nonresponders. (a) Potentials are plotted for a 1000-msec period starting $300 \mathrm{msec}$ before onset of the stationary background. Vertical gray columns mark the period of background stimulus presentation. Group means of responses under the high (bold lines) and the low (thin lines) Filehne illusion condition are overlaid for each electrode position. (b) Magnified representation of ERPs recorded in the group of Filehne-responders with labels attached to the different ERP components (exemplary electrode position: O1). (c) For the group of Filehne-responders the differences between potentials obtained under the two conditions are shown as color-coded maps. The maps were computed on the basis of an interpolation algorithm assigning a voltage to a given location that is the average of the voltages recorded from the six nearest electrode leads, linearly weighted by distance from the location at stake (Buchsbaum et al., 1982). Each component is shown for a point in time corresponding to its peak latency as observed under the high Filehne illusion condition. 
tials whose amplitudes correlated with the shift of perceived motion, the first occurring at 300 msec after stimulus onset (N300). The N300 was preceded by two ERP components (P1 and N200) that have been characterized recently by recording motion-evoked potentials under stationary visual fixation. While the $\mathrm{P} 1$ is generally thought to arise from primary visual cortex, there is convincing evidence that the N200 reflects activity in visual motion processing area V5/MT. Specifically, the N200 component has been demonstrated to be invariant over a broad range of stimulus contrast (KubovÆ Kuba, Spekreijse, \& Blakemore, 1995) but to decrease dramatically when motion adaptation occurs (Bach \& Ullrich, 1994). Using dipole source analysis, Probst and colleagues (1993) were able to localize the generator of the N200 in the region of the occipito-temporo-parietal border, which has been verified to house human MT by means of imaging techniques allowing a higher spatial resolution such as PET (Zeki et al., 1991) or functional MRI (Tootell, Reppas, Kwong, et al., 1995; Tootell, Reppas, Dale, et al., 1995).

If we agree that the N200 reflects neuronal activity in area MT, it seems reasonable to suppose that the N300 arises from a later stage in the hierarchy of cortical motion processing because it follows the N200 by 120 msec. A comparison with single-unit recordings in macaque monkeys suggests that the human homologue of monkey area MST, the next stage in the cortical processing of visual motion, might house the generator of the N300 (Erickson \& Thier, 1991; Komatsu \& Wurtz, 1988a, $1988 \mathrm{~b})$. Unlike cells in the earlier stages of motion processing, including V1 and V5/MT, which respond to retinal image slip independent of its origin, many cells in MST respond preferentially to externally induced retinal image slip but only weakly to self-induced retinal image slip (Erickson \& Thier, 1991; Ilg \& Thier, 1996). These findings suggest that the activity of these cells might be much more related to the percept of visual motion during self-motion than for instance neuronal activity in preceding area MT. Although the evidence for a relation of the N300 with area MST is admittedly circumstantial, it is clear that the $\mathrm{N} 300$ originates from parieto-occipital cortex, rostral to and excluding primary visual cortex. The N300 was followed by an increased frontal positivity (P300) and both the amplitudes of the N300 and the P300 were tightly related to the subjective percept of visual motion, rather than to visual motion on the retina or in extrapersonal space. In summary, our observations suggest that subjective perception or subjective awareness of visual motion depends on the propagation of neuronal activity in a parieto-occipito-frontal network that excludes the early stages of visual processing. These findings lend strong support to the recent hypothesis by Crick and Koch (1995), which suggests that in general visual stimuli enter awareness by the propagation of signals from extrastriate visual areas to frontal cortex.

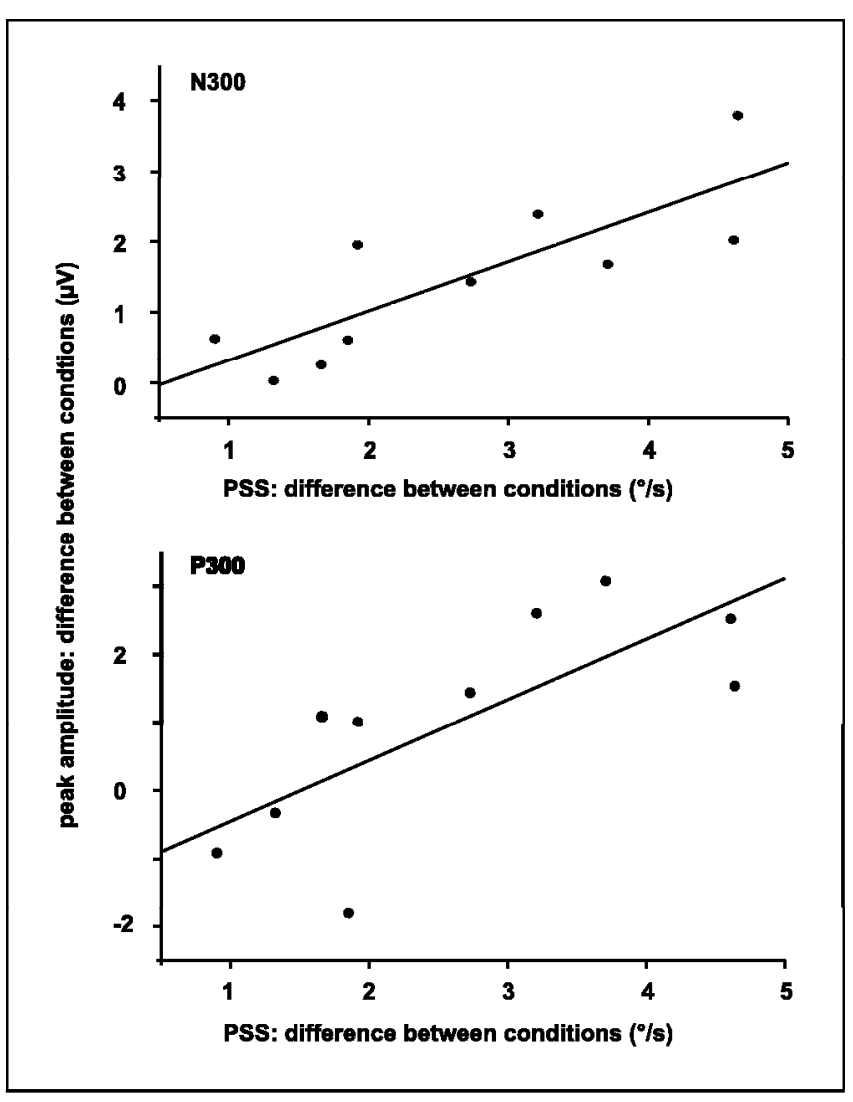

Figure 5. Peak amplitude modulation of the N300 (upper panel) and the P300 (lower panel) as a function of shift of the PSS. A linear regression is plotted both for the $\mathrm{N} 300(r=0.82)$ and the P300 $(r=$ 0.75).

\section{METHODS}

\section{Subjects}

Ten students (one female and nine males aged between 21 and 28 years) with normal visual acuity participated in this study. Subjects were right-handed and had no history of neurological disease. All gave their informed consent to participate after having the experimental protocol explained to them.

\section{Stimuli}

Stimuli were presented on a 19-in computer monitor (Mitsubishi, frame rate $72 \mathrm{~Hz}, 1280 \times 1024$ pixel) at a viewing distance of $57 \mathrm{~cm}$ in a dark experimental room. A red dot (diameter $10 \mathrm{~min}$ of arc) served as a pursuit target, which was presented for $0.5 \mathrm{sec}$ in the middle of the monitor at the beginning of each trial. The target next jumped $15^{\circ}$ to the left and then moved to the right at a constant velocity of $12^{\circ} / \mathrm{sec}$ spanning a visual angle of $30^{\circ}$ (step-ramp paradigm). Temporally located in the middle of the target sweep, a background pattern was presented for $300 \mathrm{msec}$. This background stimulus subtended $27 \times 27^{\circ}$ of visual angle and consisted of 350 white dots (diameter $15 \mathrm{~min}$ arc, local contrast, 0.01). 
The next trial was initiated $0.5 \mathrm{sec}$ after the response of the subject. ERP, PEST, and CS trials were randomly interleaved with the probability of a PEST trial being 0.5 and the probability of an ERP or conditioning trial being 0.25 each. The experiment was finished after 80 artifact-free ERP recordings.

\section{Recording of Eye Movements}

Subjects were instructed to avoid head movements and to track the pursuit target as accurately as possible. Eye movements were monitored using a head-centered infrared iris reflection system at a sampling rate of $72 \mathrm{~Hz}$. Recordings were stored and analyzed on-line by a workstation that also controlled the presentation of the stimuli and the ERP trigger. Deviations of eye position from target position exceeding $2^{\circ}$ were fed back acoustically as errors, and the corresponding trials were discarded. The means of eye velocity during background presentation were calculated separately off-line for each individual subject for the different classes of trials.

\section{Analysis of Behavioral Responses}

The PSS was defined as the background stimulus velocity that resulted in 50\% right and 50\% left responses after repeated presentation. It was determined by means of a probit analysis (McKee, Klein, \& Teller, 1985) with subsequent chi-square goodness-of-fit test performed on the responses obtained in the PEST trials.

\section{ERP Recording}

ERPs were recorded using a montage of 12 different leads with linked earlobes as reference. Ten leads were placed according to the International 10-20 system, two further leads were placed $5 \mathrm{~cm}$ left $(\mathrm{OL})$ and right (OR) of $\mathrm{Oz}$ in a fronto-parallel plane. Impedances were kept below $5 \mathrm{k} \Omega$. Signals were amplified, bandpass filtered $(0.3$ to $70 \mathrm{~Hz}$ ), and sampled with $512 \mathrm{~Hz}$. The trigger pulse started sampling $300 \mathrm{msec}$ before presentation of the stationary background, and the total sampling epoch per trial was $1000 \mathrm{msec}$. ERPs of 80 artifact-free trials were averaged in each subject, digitally low-pass filtered (cutoff frequency $20 \mathrm{~Hz}$ ), and baseline corrected on the basis of the $300-\mathrm{msec}$ interval preceding background onset.

\section{Acknowledgments}

We are grateful to Marc Repnow and Dieter Patzwahl for the development of the software we used and for many helpful discussions.

Reprint requests should be sent to P. Thier, Sektion für Visuelle Sensomotorik, Klinikum Schnarrenberg, Hoppe-SeylerStraße 3, 72076 Tübingen, Germany, or via e-mail: hanspeter.thier@uni-tuebingen.de.

\section{REFERENCES}

Bach, M., \& Ullrich, D. (1994). Motion adaptation governs the shape of motion-evoked cortical potentials. Vision Research, 34, 1541-1547.

Buchsbaum, M. S., Rigal, F., Coppola, R., Cappelletti, J., King, C., \& Johnson, J. (1982). A new system for gray-level surface distribution maps of electrical activity. Electroencephalography and Clinical Neurophysiology, 53, 237-242.

Crick, F., \& Koch, C. (1995). Are we aware of neural activity in primary visual cortex? Nature, 375, 121-123.

de Graaf, B., \& Wertheim, A. H. (1988). The perception of object motion during smooth pursuit eye movements: Adjacency is not a factor contributing to the Filehne illusion. Vision Research, 28, 497-502.

Erickson, R. G., \& Thier, P. (1991). A neuronal correlate of spatial stability during periods of self-induced visual motion. Experimental Brain Research, 86, 608-616.

Filehne, W. (1922). Über das optische wahrnehmen von bewegungen. Zeitschrift für Sinnesphysiologie, 53, 134145.

Haarmeier, T., \& Thier, P. (1996). Modification of the Filehne illusion by conditioning visual stimuli. Vision Research, 36, 741-750.

Ilg, U. J., \& Thier, P. (1996). Inability of rhesus monkey area V1 to discriminate between self-induced and externally induced retinal image slip. European Journal of Neuroscience, 8, 1156-1166.

Komatsu, H., \& Wurtz, R. (1988a). Relation of cortical areas MT and MST to pursuit eye movements. I. Localization and visual properties of neurons. Journal of Neurophysiology, 60, 580-603.

Komatsu, H., \& Wurtz, R. (1988b). Relation of cortical areas MT and MST to pursuit eye movements. III. Interaction with full-field visual stimulation. Journal of Neurophysiology, 60, 621-644.

Kubová, Z., Kuba, M., Spekreijse, H., \& Blakemore, C. (1995). Contrast dependence of motion-onset and pattern-reversal evoked potentials. Vision Research, 35, 197-205.

Mack, A, \& Herman, E. (1973). Position constancy during pursuit eye movements: An investigation of the Filehne illusion. Quarterly Journal of Experimental Psychology, 25, 71-84.

Mack, A., \& Herman, E. (1978). The loss of position constancy during pursuit eye movements. Vision Research, 18, 5562.

McKee, S. P., Klein, S. A., \& Teller, D. Y. (1985). Statistical properties of forced choice psychometric functions: Implications of probit analysis. Perception and Psychopbysics, 37 , 286-298.

Pelah, A., \& Barlow, H. B. (1996). Visual illusion from running. Nature, 381, 283.

Probst, T., Plendl, H., Paulus, W., Wist, E. R., \& Scherg, M. (1993). Identification of the visual motion area (area V5) in the human brain by dipole source analysis. Experimental Brain Research, 93, 345-351.

Rugg, M. D., Doyle, M. C., \& Well, T. (1995). Word and nonword repetition within- and across-modality: An event-related potential study. Journal of Cognitive Neuroscience, 7, 209-227.

Sperry, R. W. (1950). Neural basis of the spontaneous optokietic response produced by visual inversion. Journal of Comparative Physiology and Psychology, 43, 482-489.

Taylor, M. M., \& Creelman, C. D. (1967). PEST: Efficient estimates on probability functions. Journal of the Acoustical Society of America, 41, 782-787.

Tootell, R. B., Reppas, J. B., Dale, A. M., Look, R. B., Sereno, M. I., Malach, R., Brady, T. J., \& Rosen, B. R. (1995). Visual 
motion aftereffect in human cortical area MT revealed by functional magnetic resonance imaging. Nature, 375, 139141.

Tootell, R. B., Reppas, J. B., Kwong, K. K., Malach, R., Born, R. T., Brady, T. J., Rosen, B. R., \& Belliveau, J. W. (1995). Functional analysis of human MT and related visual cortical areas using magnetic resonance imaging. Journal of Neuroscience, 15, 3215-3230.

von Helmholtz, H. (1910). Handbuch der physiologischen Optik. Vol. 3, Leipzig: Voss.

von Holst, E., \& Mittelstaedt, H. (1950). Das Reafferenzprinzip. Naturwissenschaften, 37, 464-476.
Wertheim, A. H. (1981). On the relativity of perceived motion. Acta Psychologica, 48, 97-110.

Wertheim, A. H. (1987). Retinal and extraretinal information in movement perception: How to invert the Filehne illusion. Perception, 16, 299-308.

Wertheim, A. H. (1994). Motion perception during selfmotion: The direct versus inferential controversy revisited. Bebavioral and Brain Sciences, 17, 293-355.

Zeki, S., Watson, J. D. G., Lueck, C. J., Friston, K. J., Kennard, C., \& Frackowiak, R. S. J. (1991). A direct demonstration of functional specialization in human visual cortex. Journal of Neuroscience, 11, 641-649. 